\title{
ACM/Springer Mobile Networks and Applications (MONET) Special Issue on "Collaborative Computing: Networking, Applications and Worksharing"
}

\author{
Weisong Shi • James Joshi • Tao Zhang • Eun K. Park • \\ Juan Quemada
}

Published online: 23 May 2012

(C) Springer Science+Business Media, LLC 2012

Recent advances in computing have contributed to the growing interconnection of our world, including $3 \mathrm{G} / 4 \mathrm{G}$ wireless networks, web 2.0 technologies, computing clouds, just to mention a few. The potential for collaboration among various components has exceeded the current capabilities of traditional approaches to system integration and interoperability. As the world heads towards unlimited connectivity and global mobile computing, collaboration becomes one of the fundamental challenges. We view collaborative computing as the glue that brings the components together and also the lubricant that make them work together. The 5th International Conference on Collaborative Computing: Networking, Applications and Worksharing (CollaborateCom) serves as a premier international forum for discussion

\author{
W. Shi \\ Wayne State University, \\ Detroit, MI, USA \\ e-mail: weisong@wayne.edu \\ J. Joshi $(\square)$ \\ University of Pittsburgh, \\ Pittsburgh, PA, USA \\ e-mail: jjoshi@sis.pitt.edu \\ T. Zhang \\ Telcordia Technologies, \\ Piscataway, NJ, USA \\ e-mail: tao@research.telcordia.com \\ E. K. Park \\ University of Missouri, \\ Columbia, MO, USA \\ e-mail: ekpark@umkc.edu \\ J. Quemada \\ Universidad Politecnica De Madrid, \\ Madrid, Spain \\ e-mail: jquemada@dit.upm.es
}

among researchers and practitioners interested in collaborative networking, technology and systems, and applications.

A total of 81 submissions were received for CollaborateCom2009. The Program Committee selected 28 papers (acceptance rate of $34.6 \%$ ) after a rigorous review and a follow up discussion. After further review of the accepted papers immediately after the conference, we have selected the three papers that relates to the theme of CollaborateCom and mobile computing.

In the first paper titled "Still Alive: Extending Keep-Alive Intervals in P2P Overlay Networks," Price et al. considers the node failure detection problem within peer-to-peer networks, an inherent trade-off between timely detection and bandwidth consumption for network maintenance. Building upon several studies that have shown that older peers are more likely to remain in the network than their short-lived counterparts, the authors investigate three novel algorithms which prioritize keep-alive messages to nodes that are more likely to have failed. These algorithms significantly reduce the expected delay between failures occurring and their subsequent detection in comparison to the standard periodic approach. They also show that these algorithms can be robustly deployed in untrustworthy environments.

In the second paper titled "The Design and Evaluation of Task Assignment Algorithms for GWAP-based Geospatial Tagging Systems," Chen et al. introduce three metrics and develop five task assignment algorithms to evaluate the GWAP-based geospatial tagging systems, which are very promising for the development of advanced location-based services. Their simulation results show that the LeastThoughput-First Assignment algorithm (LTFA) is the most effective approach because it can achieve competitive system utility, while its computational complexity remains moderate. They also made several suggestions in order to achieve higher system utility. 
In the third paper titled "Secure Neighborhood Creation in Wireless Ad Hoc Networks using Hop Count Discrepancies," Hayajneh et al. study the secure neighborhood discovery problem in ad hoc and sensors networks, in which the nodes typically try to discover their neighbors simply by broadcasting a neighbor discovery request. Each node that hears the request responds with a neighbor discovery reply. This algorithm is not secure because of possible wormhole attacks. In this paper, the authors propose SECUND, a protocol for creating a secure neighborhood, that makes use of discrepancies in routing hop count information to detect "true" neighbors and remove those links to nodes that appear to be neighbors, but are not really neighbors. SECUND is very robust and effective in the presence of multiple and multi-ended wormholes.

Finally, we strongly believe that the selected papers make a significant contribution to researchers, practitioners, and students working in the areas of collaborative mobile systems and applications.

We would like to express our sincere gratitude to all the authors for their contributions and to the referees for their hard work in reviewing the papers. Our special thanks also to the editorial board of MONET, the staff at editor's office, and Prof. Imrich Chlamtac, the Editor in Chief of this journal, for their support throughout. 